\title{
Polarized and diameter-dependent Raman scattering from individual aluminum nitride nanowires: The antenna and cavity effects
}

Hsu-Cheng Hsu, Geng-Ming Hsu, Yu-shiung Lai, Zhe Chuan Feng, Shuo-Yen Tseng, Anders Lundskog, Urban Forsberg, Erik Janzén, Kuei-Hsien Chen and Li-Chyong Chen

\section{Linköping University Post Print}

N.B.: When citing this work, cite the original article.

Original Publication:

Hsu-Cheng Hsu, Geng-Ming Hsu, Yu-shiung Lai, Zhe Chuan Feng, Shuo-Yen Tseng, Anders Lundskog, Urban Forsberg, Erik Janzén, Kuei-Hsien Chen and Li-Chyong Chen, Polarized and diameter-dependent Raman scattering from individual aluminum nitride nanowires: The antenna and cavity effects, 2012, Applied Physics Letters, (101), 12, 121902.

http://dx.doi.org/10.1063/1.4753798

Copyright: American Institute of Physics (AIP) http://www.aip.org/

Postprint available at: Linköping University Electronic Press http://urn.kb.se/resolve?urn=urn:nbn:se:liu:diva-85091 


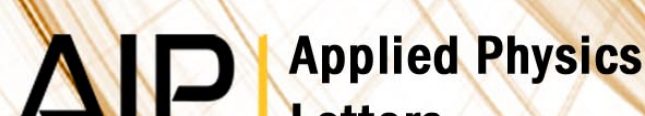 \\ Letters}

\section{Polarized and diameter-dependent Raman scattering from individual aluminum nitride nanowires: The antenna and cavity effects}

Hsu-Cheng Hsu, Geng-Ming Hsu, Yu-shiung Lai, Zhe Chuan Feng, Shuo-Yen Tseng et al.

Citation: Appl. Phys. Lett. 101, 121902 (2012); doi: 10.1063/1.4753798

View online: http://dx.doi.org/10.1063/1.4753798

View Table of Contents: http://apl.aip.org/resource/1/APPLAB/v101/i12

Published by the American Institute of Physics.

\section{Related Articles}

Can interference patterns in the reflectance spectra of GaN epilayers give important information of carrier concentration?

Appl. Phys. Lett. 101, 191102 (2012)

Influence of $p$-doping on the temperature dependence of InAs/GaAs quantum dot excited state radiative lifetime Appl. Phys. Lett. 101, 183108 (2012)

Electrical excitation and detection of magnetic dynamics with impedance matching Appl. Phys. Lett. 101, 182402 (2012)

Atomic-resolution study of polarity reversal in GaSb grown on Si by scanning transmission electron microscopy J. Appl. Phys. 112, 093101 (2012)

Dielectric strength, optical absorption, and deep ultraviolet detectors of hexagonal boron nitride epilayers Appl. Phys. Lett. 101, 171112 (2012)

\section{Additional information on Appl. Phys. Lett.}

Journal Homepage: http://apl.aip.org/

Journal Information: http://apl.aip.org/about/about_the_journal

Top downloads: http://apl.aip.org/features/most_downloaded

Information for Authors: http://apl.aip.org/authors

\section{ADVERTISEMENT}
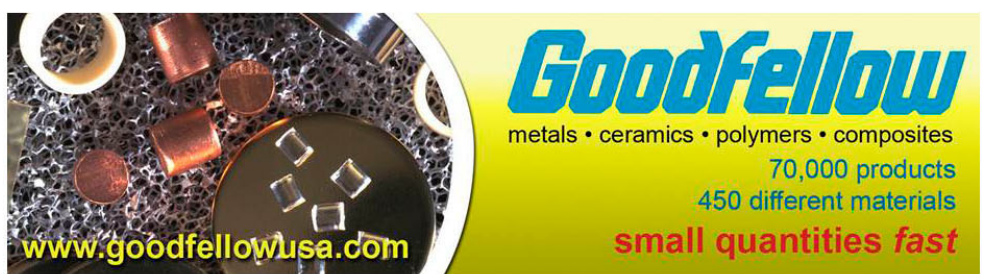


\title{
Polarized and diameter-dependent Raman scattering from individual aluminum nitride nanowires: The antenna and cavity effects
}

\author{
Hsu-Cheng Hsu, ${ }^{1}$ Geng-Ming Hsu, ${ }^{2}$ Yu-shiung Lai, ${ }^{3}$ Zhe Chuan Feng, ${ }^{3}$ \\ Shuo-Yen Tseng, ${ }^{1}$ Anders Lundskog, ${ }^{4}$ Urban Forsberg, ${ }^{4}$ Erik Janzén, ${ }^{4}$ \\ Kuei-Hsien Chen, ${ }^{2,5}$ and Li-Chyong Chen ${ }^{2, a)}$ \\ ${ }^{1}$ Department of Photonics and Advanced Optoelectronic Technology Center, National Cheng Kung University, \\ Tainan, Taiwan \\ ${ }^{2}$ Center for Condensed Matter Sciences, National Taiwan University, Taipei, Taiwan \\ ${ }^{3}$ Graduate Institute of Photonics and Optoelectronics, National Taiwan University, Taipei, Taiwan \\ ${ }^{4}$ Department of Physics, Chemistry, and Biology (IFM), Linköping University, Linköping, Sweden \\ ${ }^{5}$ Institute of Atomic and Molecular Sciences, Academia Sinica, Taipei, Taiwan
}

(Received 28 May 2012; accepted 3 September 2012; published online 17 September 2012)

\begin{abstract}
Raman scattering of individual aluminum nitride (AIN) nanowires is investigated systematically. The axial direction of single nanowire can be rapidly verified by polarized Raman scattering. The angular dependencies of $\mathrm{E}_{2}(\mathrm{high})$ mode show strongly anisotropic behavior in smaller nanowires, which results from optical antenna effect. Raman enhancement (RE) per unit volume of $E_{2}($ high) increases with decreasing diameter of nanowires. Compared to the thin film, 200-fold increase of RE is observed in AlN nanowires having diameter less than $50 \mathrm{~nm}$, which is far beyond the quantum confinement regime. Such a large RE can be attributed to the effects of resonant cavity and stimulated Raman scattering. (C) 2012 American Institute of Physics. [http://dx.doi.org/10.1063/1.4753798]
\end{abstract}

Nanostructured materials ranging from quantum dots to nanowires have recently attracted considerable attention because of the interesting physics in reduced dimension and the spatially confined electronic and optical properties. Group III-nitride is an important class of materials and has been actively sought-after for the last few decades owing to the range of properties they encompass. Among them, AlN is relatively underdeveloped, although its thin films and nanostructures have shown attractive physical and chemical properties, such as low electron affinity, strong piezoelectricity, high surface acoustic wave velocity, high thermal conductivity, and band gap tenability, which are essential for applications. A number of studies on fabrication and characterization of AlN nanostructures have been reported. ${ }^{1-7}$ Several promising applications such as field emitters, ${ }^{8-11}$ hydrogen storage media, ${ }^{12,13}$ light emitting devices, ${ }^{14-16}$ and photo detectors ${ }^{17}$ have been demonstrated. AlN nanorods also served as the barrier layer in GaN/AlN quantum dots/wire system for single photon source owing to the strong confinement of electron-hole pair as a result of its large band offset. ${ }^{18} \mathrm{In}$ addition, AlN nanowires with silver nanoparticles were used as a template of surface-enhanced Raman scattering for molecular sensing. ${ }^{19}$ Most studies are performed over an ensemble of nanostructures exhibiting a broad size distribution. Therefore, a comprehensive understanding of properties of AlN nanostructures, especially those pertinent to the reduced dimension and/or possessing size-dependence, would be extremely important.

Raman spectroscopy is a simple yet powerful technique for probing the vibration behavior of materials, which can provide information on the crystal phase, orientation, as well as lattice dynamics through a set of well-established selection rules. While the diameter of the nanowires is smaller

\footnotetext{
${ }^{\text {a) }}$ Author to whom correspondence should be addressed. Electronic mail: chenlc@ntu.edu.tw.
}

than the focus spot of the laser, the Raman scattering should be strongly affected by its diameter because the effective electric field inside the nanowire can be changed. More recently, micro-Raman studies on individual nanowires such as $\mathrm{Si}^{20}{ }^{20},{ }^{21} \mathrm{GaP},{ }^{22} \mathrm{WS}_{2},{ }^{23} \mathrm{SiC},{ }^{24} \mathrm{ZnO},{ }^{25} \mathrm{GaAs},{ }^{26} \mathrm{RuO}_{2},{ }^{27}$ $\mathrm{V}_{2} \mathrm{O}_{5},{ }^{28}$ and $\mathrm{GaN}^{29}$ demonstrate their impressive potential for probing the crystal properties of these materials. Investigation on vibration/optical properties of isolated AlN nanowires is, however, relatively rare due to a lack of mature growth technique of AlN nanowires, comparing with other semiconductor nanowires. In this report, we performed polarized micro-Raman measurements on individual AIN nanowires. The growth axis direction of single AlN nanowires can be easily determined by polarized Raman measurement. Moreover, as the diameter decreases, the Raman enhancement per unit volume increases by two orders of magnitude.

AIN nanowires were synthesized by vapor transport and condensation process. ${ }^{3,14}$ Briefly, AlN nano-structures were fabricated by vaporizing $\mathrm{Al}$ powders under ammonia $\left(\mathrm{NH}_{3}\right)$ environment in a tubular furnace by atmospheric pressure thermal chemical vapor deposition technique. Samples studied here were grown on $c$-plane (0002) sapphire instead of $\mathrm{Si}$ (100) to inhibit $\mathrm{Si}$ atom diffusion into nanowires with the growth temperature above $1000^{\circ} \mathrm{C}$. A $c$-plane sapphire substrate pre-coated with $\mathrm{Ni}$ was placed in an alumina boat, which in turn was put into the center of an alumina tube in the middle of a high-temperature cylindrical tube furnace. The furnace temperature was heated to $1500^{\circ} \mathrm{C}$ at a rate of $5^{\circ} \mathrm{C} / \mathrm{min}$ and held for a period of $180 \mathrm{~min}$. After this process, a white-colored product was obtained on the surface of the substrate. For optical study of single object, the nanowires were dispersed onto a patterned sapphire substrate with $100 \mathrm{~nm}$ thick Au film to identify and locate the positions of single wires. Optical imaging could not resolve closely spaced wire pairs, so field emission scanning electron microscopy (FESEM) was used independent of the optical 
setup to image the samples of interest. More than 50 isolated nanowires and tapered nanowires were examined.

Raman spectra were recorded at room temperature in back-scattering geometry, using a micro-Raman spectrometer (LabRAM HR 800 UV, Jobin Yvon) with a charge-coupled device (CCD) detector and an adjustable notch filter. The sample was excited by the $632.8 \mathrm{~nm}$ line of a He-Ne laser. A confocal microscope (BX41, Olympus) was equipped with a $100 \times$ objective which gives laser spot about $1 \mu \mathrm{m}$ on the sample surface. To minimize the heating effect from laser, the laser power was reduced to low power $(<1 \mathrm{~mW})$ by a neutral density filter. No significant broadening, peak shift, or decrease in intensity was observed during data acquisition, indicating thermal heating effect can be ruled out.

Spectra were collected while controlling polarization of both incident and scattered radiations. To simplify the measurement and the transformation from laboratory frames to crystal-fixed frames, the origin of laboratory-fixed frames was chosen so that the long axis of the nanowire would lie along one of the canonical lab directions (the $\mathrm{X}$ direction). Likewise, the crystal-fixed frames were defined accordingly such that the $\mathrm{x}, \mathrm{y}, \mathrm{z}$ axes were aligned along the [1010], [1210], [0002] crystallographic directions. In this geometry, we aligned our nanowires along the laboratory Y-axis and measured the polarization-dependent Raman intensity with the incident and scattered light polarization rotating from the $\mathrm{Y}$-axis (parallel) to the $\mathrm{X}$-axis (perpendicular) keeping both incident and scattering light polarized in the same direction. For the measurement of individual AlN nanowires, three different polarization configurations, namely, $X(Y Y) \bar{X}, X(Z Z) \bar{X}$, and $X(Y Z) \bar{X}$ (according to the Proto notation) were used, where the c-axis of the nanowire is defined as the $\mathrm{Z}$-axis. Porto's notation $A(B C) D$ is used to describe the Raman geometry and polarization, where $A$ and $D$ represent the wavevector direction of the incoming and the scattered light, respectively, while $B$ and $C$ represent the polarization direction of the incoming and scattered light. The identification of all phonon modes can be performed by comparing polarized Raman scattering studies with the theoretical predictions obtained through the Raman tensors. ${ }^{30}$ The space group of wurtzite AlN is $C_{6 v}^{4}$ and the Raman active modes are $1 \mathrm{~A}_{1}+1 \mathrm{E}_{1}+2 \mathrm{E}_{2}$, while $2 \mathrm{~B}_{2}$ modes are silent modes. Since the wurtzite structure is noncentrosymmetric, both $\mathrm{A} 1$ and E1 mode split into longitudinal optical (LO) and transverse optical (TO) components.

First, the structure characterizations of ensemble were examined. X-ray diffraction (XRD) analysis was performed to investigate the crystal structure of the products. As shown in Fig. 1(a), the XRD patterns revealed that all the diffraction peaks can be indexed as hexagonal wurtzite structure with cell constants of $a=0.311 \mathrm{~nm}$ and $c=0.498 \mathrm{~nm}$. No excess peaks could be detected, except for peak from sapphire substrate, which indicates that no other phases were formed. The Raman spectrum of as-grown AlN nanowires is illustrated in Fig. 1(b). Four distinct peaks at 249, 611, 657, $671 \mathrm{~cm}^{-1}$ were observed, which can be identified to $\mathrm{E}_{2}(\mathrm{low})$, $A_{1}(\mathrm{TO}), \mathrm{E}_{2}$ (high), $\mathrm{E}_{1}(\mathrm{TO})$, respectively. The broad peak at $895 \mathrm{~cm}^{-1}$ is related to the overlap of the $\mathrm{A}_{1}(\mathrm{LO})$ and $\mathrm{E}_{1}(\mathrm{LO})$ modes. These results are in agreement with those reports of AlN nanostructures ${ }^{15,31}$ as well as films. ${ }^{32}$
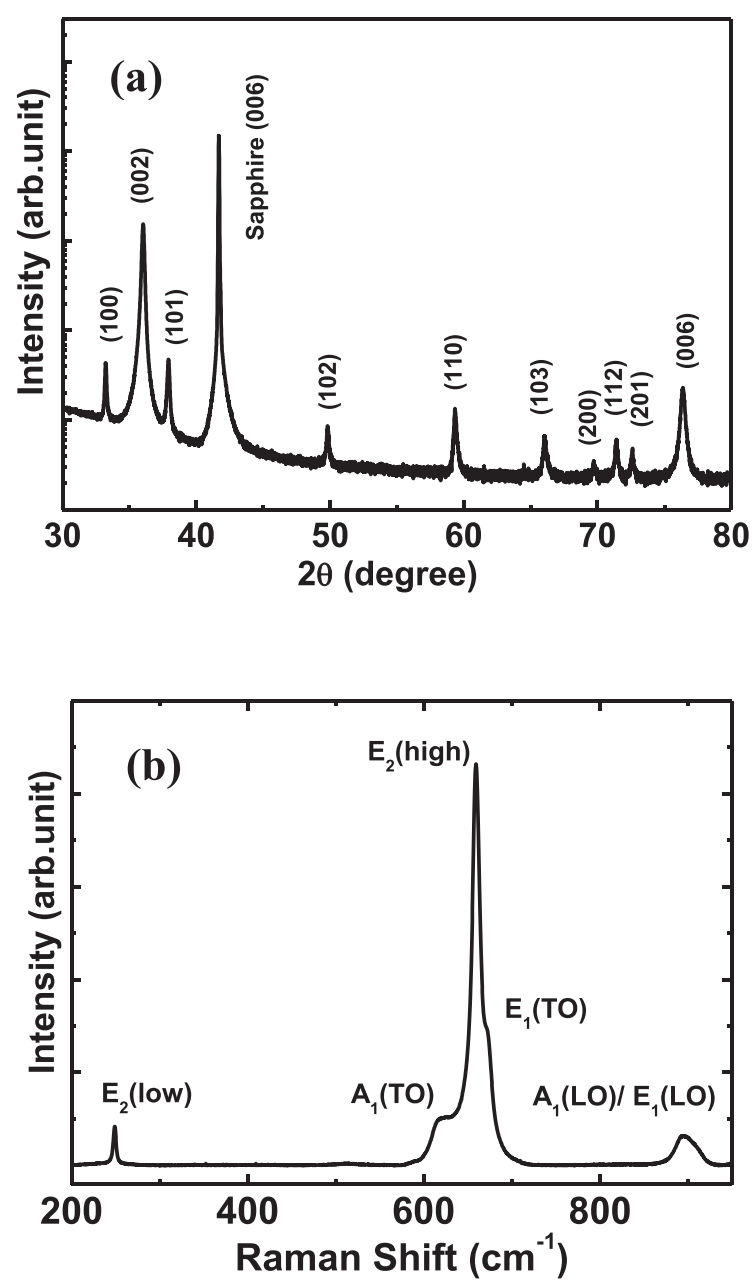

FIG. 1. (a) XRD pattern of ensemble AIN nanowires. (b) Micro-Raman scattering spectrum of ensemble AlN nanowires.

Steps to determining the growth direction of isolated AlN nanowires are illustrated as following. Shown in Fig. 2(a) is a scanning electron microscopy image of a typical AlN nanowire under investigation. The mean diameter and length of this nanowire are $450 \mathrm{~nm}$ and $7 \mu \mathrm{m}$, respectively. Figure 2(b) shows typical Raman spectra taken under different polarized configurations from this AlN nanowire. Strong polarization dependence can be observed. In the $X(Y Y) \bar{X}$ configuration, as in the lowest trace of Fig. 2(b), the $\mathrm{E}_{2}$ (high) mode is predominant and a weak $\mathrm{A}_{1}(\mathrm{TO})$ modes could be observed. In the $X(Y Z) \bar{X}$ configuration, all the $\mathrm{A}_{1}(\mathrm{TO}), \mathrm{E}_{2}(\mathrm{high})$, and $\mathrm{E}_{1}(\mathrm{TO})$ modes appear. While the polarized configuration is changed to $X(Z Z) \bar{X}$ configuration, the $\mathrm{A}_{1}(\mathrm{TO})$ mode becomes dominant and a weak intensity of $E_{1}(T O)$ could be observed. The observation of non-vanished modes of $\mathrm{A}_{1}(\mathrm{TO})\left(\mathrm{E}_{2}\right.$ (high)) in $X(Y Z) \bar{X}(X(Z Z) \bar{X})$, which are forbidden in corresponding geometries, should be due to reflections inside the crystal and/or a small tilting of the sample. All the Raman modes could be used for identification of growth orientation except for these above mentioned non-zero modes. As a result, we can conclude that the $c$-axis is parallel to the long axis of nanowire according to theoretical predictions. Figure 2(c) plots the intensities of Raman modes as a function of the angle between the polarization direction of the incident laser and the long axis of nanowire. The trends of intensities for each mode are in good accord with the calculated results of wurtzite structure. ${ }^{27}$ 

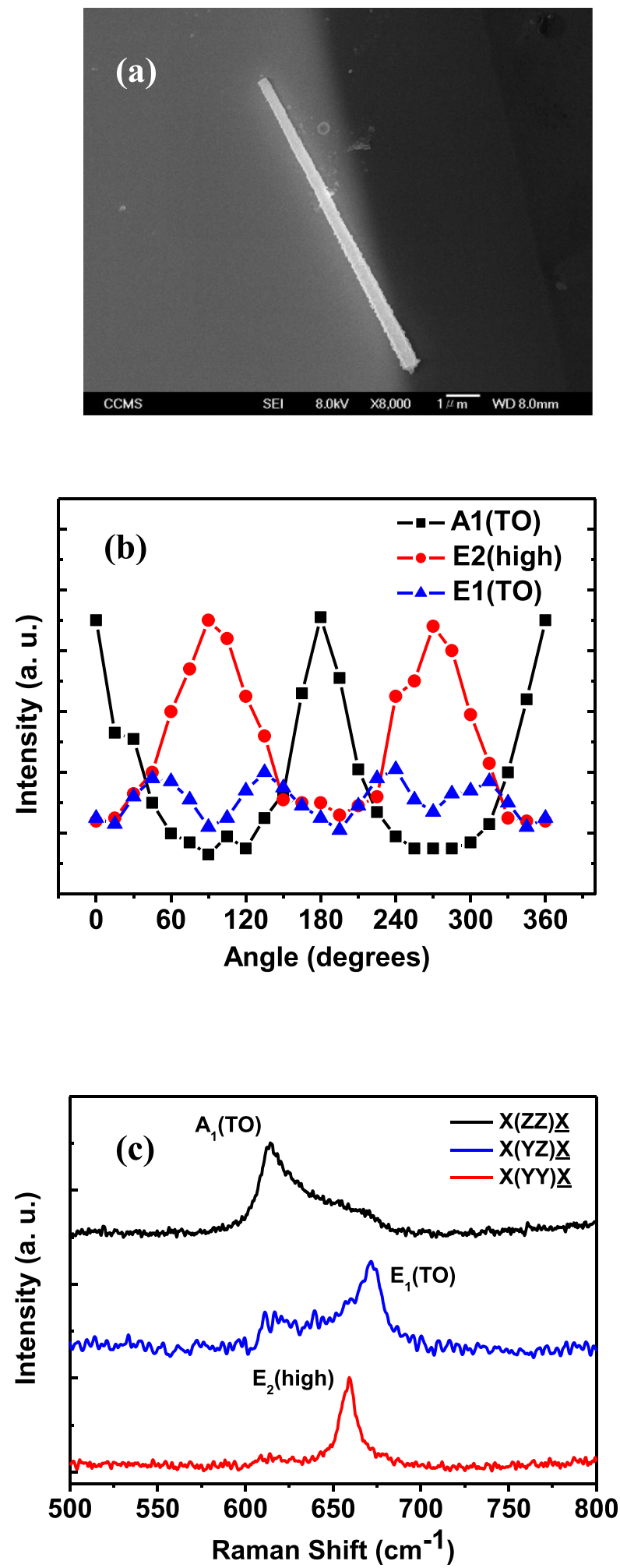

FIG. 2. (a) Scanning electron microscopy image of a single AIN nanowire. (b) Typical micro-Raman scattering spectra of individual $c$-axis oriented AlN nanowire with different polarization configurations. (c) Intensities of the Raman modes as a function of the angle between the polarization direction of the incident laser and the long axis of nanowire.

Besides AIN nanowires with $c$-axis growth direction, AlN nanowires with other orientation direction were also observed. In different polarization configurations, the three peaks $-\mathrm{E}_{2}$ (high), $\mathrm{A}_{1}(\mathrm{TO})$, and $\mathrm{E}_{1}(\mathrm{TO})$ can be observed and the relative intensities of each mode are identical in Fig. 3. According to Raman selection rules, the c-axis is perpendic-

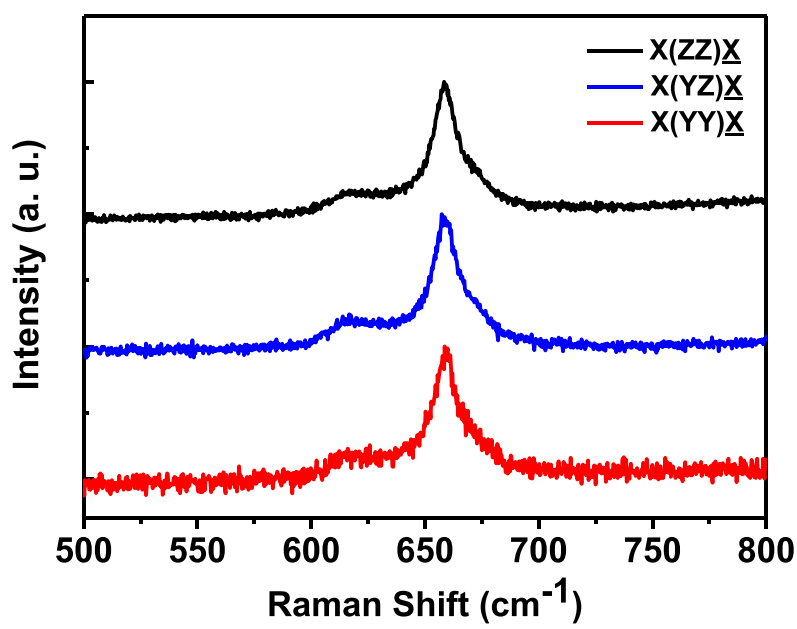

FIG. 3. Typical micro-Raman scattering spectra of individual non-c-axis oriented AlN nanowire with different polarization configurations.

ular to the long axis of the nanowire. The apparent polarization dependence of Raman modes observed here is quite similar with the results of the observation of GaN nanowires. ${ }^{24-26}$ Although the mechanism of growth direction for nanowires is not yet well understood, we still could use Raman scattering as a powerful tool to identify the growth direction of nanowires.

Polarized direction-dependent Raman spectra were studied in details, especially for the $\mathrm{E}_{2}$ (high) mode because of its characteristic phonon mode. Figure 4 shows the polar patterns of $\mathrm{E}_{2}$ (high) mode of three different typical $c$-axis nanowires with $237 \mathrm{~nm}, 120 \mathrm{~nm}$, and $42 \mathrm{~nm}$ in diameter. For wire with a larger diameter, the Raman scattering intensities are observed to exhibit a $\cos ^{4} \theta$ (dash line) response, where $\theta$ is the angle between the incident laser direction and the nanowire axis, as expected in the calculated result of wurtize-type nanorods. ${ }^{27}$ Interestingly, for wire with a smaller diameter, the trend of Raman intensities cannot be fitted by the function of $\cos ^{4} \theta$ well and should be fitted by higher exponential term. This is likely due to the Raman antenna effect which has been observed in isolated single-wall carbon nanotubes (SWNTs), $\mathrm{WS}_{2}$ nanotubes, and GaP nanowires. ${ }^{20}$ Our results

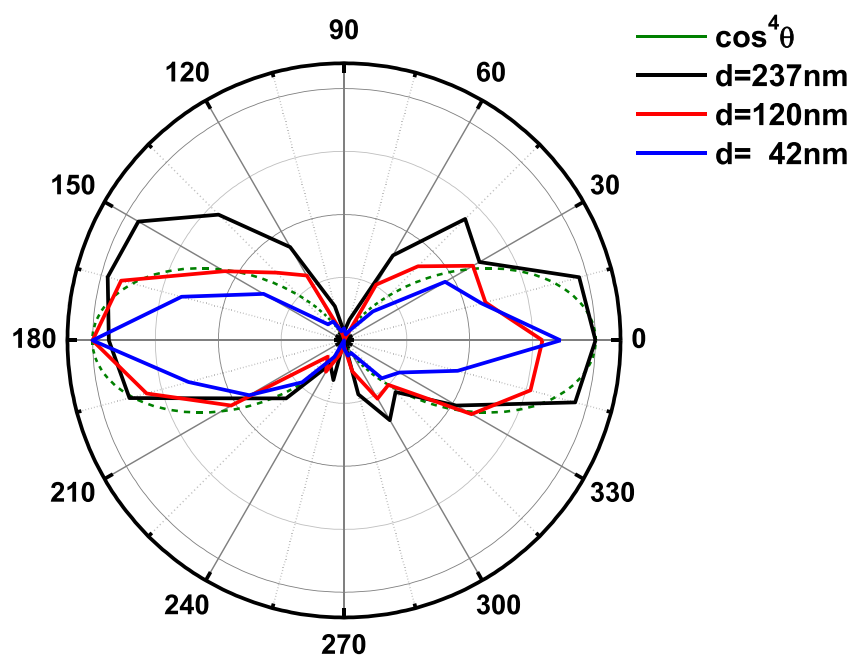

FIG. 4. Polar plots showing the Raman mode of $E_{2}$ (high) of AlN nanowires as a function of the angle between the polarization of the incident light and the nanowire axis. 
suggest that AlN nanowires with small diameter may be served as optical antenna photovoltaic absorbers ${ }^{33}$ and/or gain media for Raman lasers. ${ }^{34}$

Furthermore, we performed diameter-dependent Raman of individual $c$-axis AlN nanowires and monitored the intensities of $E_{2}$ (high) mode. The peak position and full width at half maximum (FWHM) of $\mathrm{E}_{2}$ (high) mode do not obviously change as a function of diameter (not shown here). Since the diameters of the studied AlN nanowires are ranging from 33 to $4000 \mathrm{~nm}$, which are significantly larger than the Bohr exciton radius in $\mathrm{AlN}$, no quantum confinement effects are observed.

To make a comparison of Raman enhancement, we define the value of Raman enhancement per volume (RE) as follows: ${ }^{20}$

$$
R E=\frac{I_{n w} / V_{n w}}{I_{r e f} / V_{r e f}},
$$

where $I_{n w}$ represents the measured Raman intensity, $I_{\text {ref }}$ is for the high quality thin film. ${ }^{35} \mathrm{~V}_{\mathrm{nw}}$ and $\mathrm{V}_{\text {ref }}$ denote the volume of nanowire and thin film, respectively. Although the intensity of Raman decreases with decreasing diameter, the corresponding RE is relatively significant.

A cavity phenomenon involving incident electromagnetic radiation and the structure dielectric cross section was proposed to explain the observed Raman enhancement in $\mathrm{Si}$ nanowires. ${ }^{20}$ We also model the light scattering by solving Maxwell's equations using complex index of refraction of AlN, following the approach reported in Ref. 20. The calculated values of RE are compared with the experimental data in Figure 5. The trend of the value increases as diameter decreases, which is in good agreement with the modeling except for the case of diameters smaller than $50 \mathrm{~nm}$. The origin of enhancement may result from structural resonances in the local field similar to Mie scattering from dielectric sphere. ${ }^{36}$ For the smallest nanowires of $33 \mathrm{~nm}$ in diameter, the RE value is two orders of magnitude larger than that of films, slightly higher than that of the simulation. The discrepancy may be caused by the occurrence of the stimulated

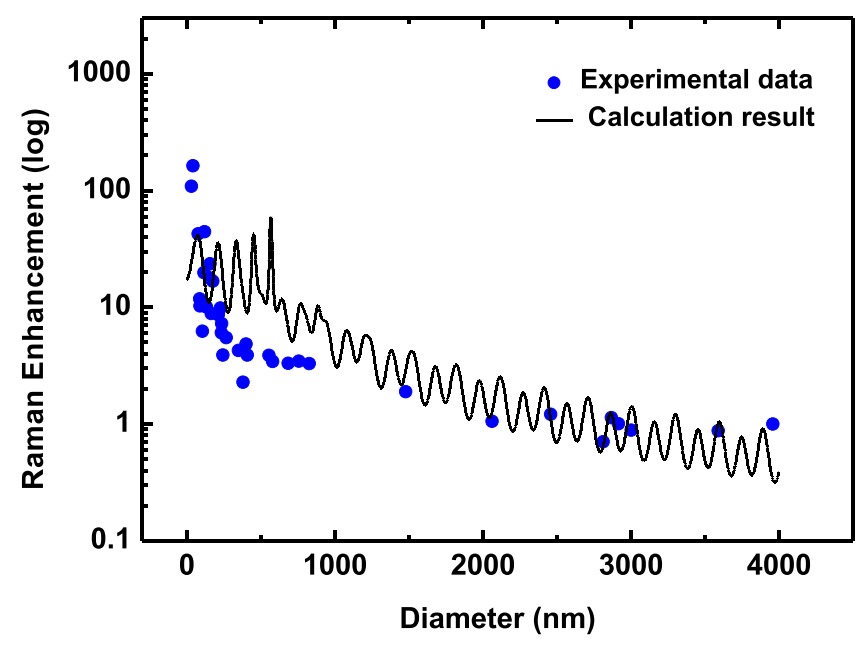

FIG. 5. The blue dots and line represent the measured Raman enhancement of $\mathrm{E}_{2}$ (high) mode for a series of $\mathrm{AlN}$ nanowires as a function of diameter under $\mathrm{X}(\mathrm{YY}) \mathrm{X}$ configuration and simulation result, respectively.
Raman scattering. ${ }^{34}$ Further refinement of the model should be performed in future.

We therefore believe the structure resonant effect should play an important role in our system. The Raman enhancement factor of AlN wires is comparable to that of Si nanowires. ${ }^{20}$ The energy of excitation laser we used is much lower than the bandgap of AlN and thus belongs to offresonant Raman condition. If one can use the laser having energy close to the bandgap of materials, it is expected to gain stronger enhancement because the scattering cross section is proportional to $\lambda^{-4}$.

In conclusion, we described the use of polarized Raman spectroscopy as a rapid and nondestructive probe for determining the phase as well as the growth orientation of AlN nanowires. We have investigated the polarized- and sizedependent characteristics of Raman scattering of individual AlN nanowires. The diameter effect plays a dominant role in strong anisotropic Raman effect of single nanowires. As the diameter of nanowire is decreased, Raman enhancement per unit volume of $E_{2}$ (high) mode increases to 2 orders of magnitude. This information could provide insight for further design of nanowire-based devices.

This work was financially supported by Ministry of Education, National Science Council and Academia Sinica in Taiwan as well as the US AFOSR-AOARD.

${ }^{1}$ Q. Wu, Z. Hu, X. Wang, Y. Chen, and Y. Lu, J. Phys. Chem. B 107, 9726 (2003).

${ }^{2}$ Q. Zhao, H. Z. Zhang, X. Y. Xu, Z. Wang, J. Xu, D. P. Yu, G. H. Li, and F. H. Su, Appl. Phys. Lett. 86, 193101 (2005).

${ }^{3}$ S. C. Shi, S. Chattopadhyay, C. F. Chen, K. H. Chen, and L. C. Chen, Chem. Phys. Lett. 418, 152 (2006); S. C. Shi, C. F. Chen, S. Chattopadhyay, Z. H. Lan, K. H. Chen, and L. C. Chen, Adv. Funct. Mater. 15, 781 (2005).

${ }^{4}$ G. R. Yazdi, M. Syväjärvi, and R. Yakimova, Appl. Phys. Lett. 90, 123103 (2007).

${ }^{5}$ H. M. Fan, X. F. Fan, Z. H. Ni, Z. X. Shen, Y. P. Feng, and B. S. Zou, J. Phys. Chem. C 112, 1865 (2008).

${ }^{6}$ F. Zhang, Q. Wu, X. Wang, N. Liu, J. Yang, Y. Hu, L. Yu, X. Wang, Z. Hu, and J. Zhu, J. Phys. Chem. C 113, 4053 (2009).

${ }^{7}$ L. Yu, Y. Lv, P. Liu, and X. Yu, Mater. Lett. 65, 1499 (2011).

${ }^{8}$ J. H. He, R. Yang, Y. L. Chueh, L. J. Chou, L. J. Chen, and Z. L. Wang, Adv. Mater. 18, 650 (2006).

${ }^{9}$ Q. Zhao, J. Xu, X. Y. Xu, Z. Wang, and D. P. Yu, Appl. Phys. Lett. 85, 5331 (2004).

${ }^{10}$ Y. B. Tang, H. T. Cong, Z. G. Zhao, and H. M. Cheng, Appl. Phys. Lett. 86, 153104 (2005).

${ }^{11}$ S. C. Shi, C. F. Chen, S. Chattopadhyay, K. H. Chen, and L. C. Chen, Appl. Phys. Lett. 87, 073109 (2005).

${ }^{12}$ Q. Wang, Q. Sun, P. Jena, and Y. Kawazoe, ACS Nano 3, 621 (2009).

${ }^{13}$ Y. F. Li, Z. Zhou, P. W. Shen, S. B. Zhang, and Z. F. Chen, Nanotechnology 20, 215701 (2009).

${ }^{14}$ S. C. Shi, C. F. Chen, S. Chattopadhyay, K. H. Chen, B. W. Ke, L. C. Chen, L. Trinkler, and B. Berzina, Appl. Phys. Lett. 89, 163127 (2006).

${ }^{15}$ W. W. Lei, J. Zhang, D. Liu, P. W. Zhu, Q. L. Cui, and G. T. Zou, Chem. Commun. 5221 (2008).

${ }^{16}$ B. Berzina, L. Trinkler, D. Jakimovica, V. Korsaks, J. Grabis, I. Steins, E. Palcevskis, S. Bellucci, L. C. Chen, S. Chattopadhyay, and K. H. Chen, J. Nanophotonics 3, 031950 (2009).

${ }^{17}$ H. M. Huang, R. S. Chen, H. Y. Chen, T. W. Liu, C. C. Kuo, C. P. Chen, H. C. Hsu, L. C. Chen, K. H. Chen, and Y. J. Yang, Appl. Phys. Lett. 96, 062104 (2010).

${ }^{18}$ J. Renard, R. Songmuang, C. Bougerol, B. Daudin, and B. Gayral, Nano. Lett. 7, 2092 (2008).

${ }^{19}$ S. Chattopadhyay, S. C. Shi, Z. H. Lan, C. F. Chen, K. H. Chen, and L. C. Chen, J. Am. Chem. Soc. 127, 2820 (2005).

${ }^{20}$ L. Cao, B. Nabet, and J. E. Spanier, Phys. Rev. Lett. 96, 157402 (2006). 
${ }^{21}$ Y. Ogawa, Y. Yuasa, F. Minami, and S. Oda, Appl. Phys. Lett. 99, 053112 (2011).

${ }^{22}$ G. Chen, J. Wu, Q. Lu, H. R. Gutierrez, Q. Xiong, M. E. Pellen, J. S. Petko, D. H. Werner, and P. C. Eklund, Nano Lett. 8, 1341 (2008).

${ }^{23}$ P. M. Rafailov, C. Thomsen, K. Gartsman, I. Kaplan-Ashiri, and R. Tenne, Phys. Rev. B 72, 205436 (2005).

${ }^{24}$ J. Frechette and C. Carraro, Phys. Rev. B 74, 161404 (2006).

${ }^{25}$ C. T. Chien, M. C. Wu, C. W. Chen, H. H. Yang, J. J. Wu, W. F. Su, C. S. Lin, and Y. F. Chen, Appl. Phys. Lett. 92, 223102 (2008).

${ }^{26}$ I. Zardo, S. Conesa-Boj, F. Peiro, J. R. Morante, J. Arbiol, E. Uccelli, G. Abstreiter, and A. F. I. Morral, Phys. Rev. B 80, 245324 (2009).

${ }^{27}$ M. H. Kim, J. M. Baik, S. J. Lee, H. Y. Shin, J. Lee, S. Yoon, G. D. Stucky, M. Moskovits, and A. M. Wodtke, Appl. Phys. Lett. 96, 213108 (2010).

${ }^{28}$ B. Yan, C. L. Du, L. Liao, Y. M. You, H. Cheng, Z. X. Shen, and T. Yu, Appl. Phys. Lett. 96, 073105 (2010).

${ }^{29}$ P. J. Pauzauskie, D. Talaga, K. Seo, P. D. Yang, and F. Lagugne-Labarthet, J. Am. Chem. Soc. 127, 17146 (2005); T. Livneh, J. Zhang, G. Cheng, and
M. Moskovits, Phys. Rev. B 74, 035320 (2006); H. Ji, M. Kuball, R. A. Burke, and J. M. Redwing, Nanotechnology 18, 445704 (2007); C. L. Hsiao, L. W. Tu, T. W. Chi, M. Chen, T. F. Young, C. T. Chia, and Y. M. Chang, Appl. Phys. Lett. 90, 043102 (2007).

${ }^{30}$ T. Azuhata, T. Sola, K. Suzuki, and S. Nakamura, J. Phys.: Condens. Matter 7, L129 (1995).

${ }^{31}$ S. Sahoo, S. Dhara, A. K. Arora, R. Krishnan, P. Chandramohan, and M. P. Srinivasan, Appl. Phys. Lett. 96, 103113 (2010).

${ }^{32}$ T. Prokofyeva, M. Seon, J. Vanbuskirk, M. Holtz, S. A. Nikishin, N. N. Faleev, H. Temkin, and S. Zollner, Phys. Rev. B 63, 125313 (2001).

${ }^{33}$ L. Cao, P. Fan, A. P. Vasudev, J. S. White, Z. Yu, W. Cai, J. A. Schuller, S. Fan, and M. L. Brongersma, Nano Lett. 10, 439 (2010).

${ }^{34}$ J. Wu, A. K. Gupta, H. R. Gutierrez, and P. C. Eklund, Nano Lett. 9, 3252 (2009).

${ }^{35}$ A. Kakanakova-Georgieva, R. R. Ciechonski, U. Forsberg, A. Lundskog, and E. Janzen, Cryst. Growth Des. 9, 880 (2009).

${ }^{36}$ G. Mie, Ann. Phys. 25, 377 (1908). 